\title{
Dehydration affects exercise-induced asthma and anaphylaxis
}

\author{
Kwi-Baek Kim', Yi-Sub Kwak,** \\ ${ }^{1}$ Youngsan Health Science Institute, Department of Marine Leisure and Tourism, Youngsan University, Busan, Korea \\ ${ }^{2}$ DEU Exe-Physio Lab, Department of Physical Education, Dong-Eui University, Busan, Korea
}

\begin{abstract}
Allergies are generally triggered by food, medication, physical exercise, stress, alcohol consumption, and dehydration. There are reports that indicate dehydration affects various kinds of physical allergies. However, there are few studies that have focused on the effects of dehydration on asthma and allergy anaphylaxis. Therefore, we analyzed the effects of dehydration on several kinds of allergy responses and exercise-induced asthma especially during the endurance exercise. PubMed was searched from April to July of 2019 using predefined search terms "dehydration," "exercise," and "allergy responses." Based on the reference search, more than one-hundred articles were identified but eighteen papers met the inclusion criteria and were analyzed for connections among exercise and dehydration, dehydration and exercise-induced asthma, and allergy responses in the main text. Results suggest that dehydration directly impairs stroke volume, cardiac output, and skin blood flow. This results in larger increases in core
\end{abstract}

\section{INTRODUCTION}

Severe hyperpnea and some agents such as exercise, nitro oxide, diesel exhaust particles, gases, irritant particles induce airway hyperresponsiveness and exercise-induced bronchoconstriction in many athletes. These agents also initiate epithelial injury due to poorly conditioned breathing during strenuous exercise. Additionally, repeated hyperpnea during exercise training or sport events activate exercise-induced asthma (EIA). It is also a related mechanism with the airway dehydration. Exercise mode, exercise duration, and severity are closely connected to water contents of inspired air and ventilation volume during physical exercise (Anderson and Kippelen, 2008).

Being in a dehydrated state during exercise is one of the main temperature, heart rate, and stroke volume. Additionally, exercise-induced dehydration reduces airway surface hydration, which results in an amplified brocnchoconstriction. This response to exercise occurs in those who suffer from exercise-induced asthma. Moreover, damage to the gut and impaired gut function relates to increased intestinal permeability after endurance exercise. Endurance exercise changes the immunological profiles to activate antibody-mediated immunity. Also, numerous mast cells and eosinophils were recruited, therefore isotype switching to IgE antibodies occur, this hypersensitivity activates mast cell degranulation. After degranulation, proteases, leukotrienes, prostaglandins, and histamine lead to many kinds of allergy symptoms.

Keywords: Dehydration, Intestinal immunity, Bronchoconstriction, Isotype switching, Anaphylaxis
${ }^{*}$ Corresponding author: Yi-Sub Kwak (ib https://orcid.org/0000-0003-4545-7250 Department of Physical Education, College of Arts, Design and Sports Science, Dong-Eui University, 176 Eomgwang-ro, Busanjin-gu, Busan 47340, Korea E-mail: ysk2003@deu.ac.kr

Received: August 15, 2019 / Accepted: September 9, 2019 causes of gastrointestinal (GI) complaints. GI complaints occur from the redistribution of the blood flow, which is shunted from viscera to liver, heart, and many skeletal muscle (Qamar and Read, 1987).

Gut ischemia is the main cause of vomiting, intestinal clamps, dizziness, diarrhea, and even nausea (de Oliveira and Burini, 2009). It also activates food allergy, allergy anaphylaxis, and food dependent exercise-induced anaphylaxis (FDEIAn). Anaphylaxis is observed during and after exercise when preceded by the intake of a causative allergen foods or some medication (Kim and Kwak, 2004; Kim et al., 2013). Hyperthermia, hypernatremia, and GI problems, are common symptoms inmarathoners and triathletes (Jeukendrup et al., 2005). Intestinal IgA depletion is correlated with increased intestinal permeability after endurance exercise. This facilitates allergen absorption from the GI tract, leading to 
food allergy and FDEIAn.

Allergies in general are initiated by several factors such as food, medication, physical exercise, stress, alcohol consumption, and dehydration. There are some reports that indicate dehydration affects various kinds of physical allergies. However, there are few studies about the effects of dehydration on asthma and allergy anaphylaxis. Therefore, we analyzed the effects of dehydration on several types of allergy responses, including EIA during and after endurance exercise.

\section{EXERCISE-INDUCED DEHYDRATION MAY INCREASE ASTHMA AND ALLERGY RESPONSES}

It is well known that dehydration directly or indirectly impairs stroke volume, cardiac output, and skin blood flow, which results in larger increases in core temperature, heart rate, and stroke volume (Coyle and Montain, 1992). Therefore, 30-60 g of carbohydrate supplementation with water during each hour of exercise will be enough to maintain exercise performance and blood glucose level. It is well reported that inadequate fluid consumption impairs the cardiovascular system. Therefore, fluid consumption must match at least $80 \%$ of sweat rate. For ultraendurance athletes, who race longer than $8 \mathrm{hr}$, dehydration and hyponatremia are reoccurring problems. Accordingly, ultraendurance athletes must understand and maintain proper fluid consumption and electrolyte balance (Hiller, 1989; Park and Kwak, 2019; Park et al., 2014). Hyponatremia symptoms, like those of dehydration, may be dangerously misunderstood by many ultraendurance athletes. Usually, mental confusion and fainting are seen at $126-130 \mathrm{mmol} / \mathrm{L}$. Below this level, coma and seizures may occur (Jeukendrup et al., 2005). Drinking adequate amounts of water is recommended for competing athletes to avoid hyponatremia. Recent research has reported that the airway response to dry air (hyperpnea) in athletes with mild asthma is not exacerbated in a state of mild dehydration (Simpson et al., 2017). However, there are many research papers that reveal the risk factor of bronchopulmonary disorders in the state of dehydration (Kalhoff, 2003; Rundell et al., 2015).

Exercise-induced dehydration reduces airway surface hydration, resulting in amplified bronchoconstriction. This is a response to exercise in EIA individuals and asthma patients. Exercise-induced dehydration alters and even damages the airway and pulmonary function, but does not modify airway responsiveness to dry air in athletes with mild asthma. This paper reported that most trainers set $2.3 \%$ body mass loss using summer physical activity and training. However, this mild degree of dehydration may not have interfered with the exercise-induced bronchoconstriction pathophysiology (Simpson et al., 2017). Though small volumes of water are available at the airway surface, airway water loss during hyperpnea of dry air is very high according to the mathematical modeling of heat and water transport in human respiratory track (Daviskas et al., 1990). Calculated local heat and water transfer rates may help to predict the area and intensity of EIA. Excessive water and heat loss from the airways aggravate asthma symptoms during and after exercise (Daviskas et al., 1991). During exercise, the temperature along the airways gradually decrease as air flow from the lung periphery to the mouth. The more drying and cooling that transpires, the lower the temperature occurred during expiration. Consequently, the fall of forced expiratory volume in 1 $\sec \left(\mathrm{FEV}_{1}\right)$ after dry air hyperpnea could worsen following endurance exercise-induced dehydration.

It is generally known that cold and dry air deteriorate EIA to protect lung function. Rapid fluid delivery is importantly considered in order to protect bronchial and lung function. It is also important to notify athletes about the dangers of drinking too much water. Cromolyn sodium supplementation modifies respiratory heat exchange and therefore limits airway cooling (Pichurko et al., 1984). This kind of treatment may produce a direct or indirect impact on the bronchial vasculature. There is also the possibility that dehydration induces FDEIAn. FDEIAn generally occurs when food allergen and physical exercise are combined, a subtype of anaphylaxis. Allergen symptoms are elicited when triggering factors such as exercise, some medication, and alcohol consumption (Fig. 1) occurs. Damage to the gut and impaired gut function is correlated with increased intestinal permeability after endurance exercise. Endurance exercise changes the immunological profiles to activate

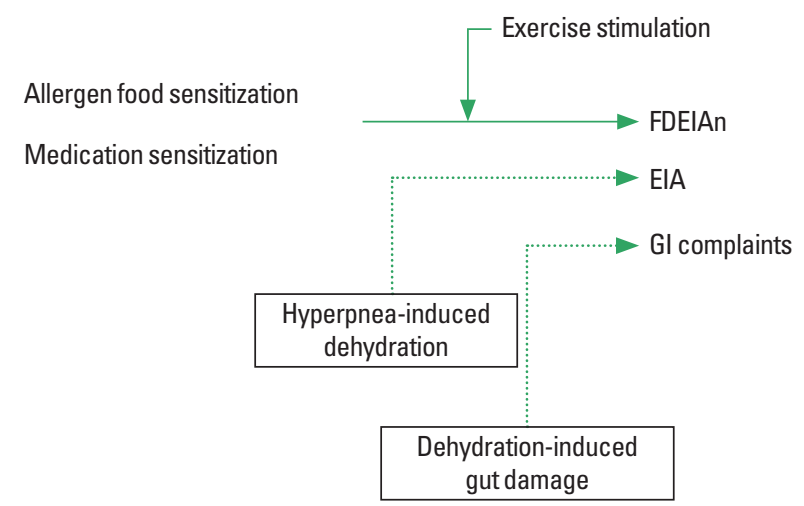

Fig. 1. Dehydration is a crucial factor in allergy responses. FDEIAn, food dependent exercise-induced anaphylaxis; EIA, exercise-induced asthma; Gl, gastrointestinal. 
antibody-mediated immunity (Fig. 1). A plethora of mast cells and successive eosinophils were also recruited, Isotype switching to IgE antibodies, IgE antibodies mediated hypersensitivity activates mast cell degranulation. After degranulation, proteases, leukotrienes, prostaglandins, and histamines lead to many allergy symptoms.

It is well reported that mechanisms redistribute blood flow to central tissues make changes in nutrient absorption, motility, and the mucosal integrity of the GI track. This results in GI complaints (Van Nieuwenhoven et al., 2000). Serious gut under perfusion often leads to shock-induced mucosal damage and invasion of intestinal bacteria or endotoxins into blood circulation (Van Deventer and Gouma, 1994). Gut mucosa is susceptible to ischemic injury, increases mucosa permeability and endotoxin generation. This also initiates mucosal inflammation and even cell death. Further mechanism studies that focus on the impact of dehydration (with diverse exercise) on intestinal immunity is urgently needed in this area.

\section{CONCLUSIONS}

There are few studies about the effects of dehydration on asthma and allergy anaphylaxis, despite dehydration being an important factor for allergy responses and intestinal immunity. Therefore, we analyzed the dehydration effects on several kinds of allergy responses and even exercise-induced asthma during endurance exercise. Results suggest that dehydration directly or indirectly impairs stroke volume, cardiac output, and skin blood flow, which results in larger increases in core temperature, heart rate and etc. Exercise-induced dehydration reduces airway surface hydration, resulting in increased bronchoconstriction. This is a response to exercise in EIA individuals and asthma patients.

Moreover, damage to the gut and impaired gut function is correlated with increased intestinal permeability after endurance exercise. Endurance exercise changes the immunological profiles to activate antibody-mediated immunity. Heaps of mast cells and eosinophils were recruited, Isotype switching to $\operatorname{IgE}$ antibodies occur, therefore hypersensitivity activates mast cell degranulation. Further mechanism studies that focus on the impact of dehydration (with diverse exercise) on intestinal immunity is urgently needed in this area.

\section{CONFLICT OF INTEREST}

No potential conflict of interest relevant to this article was reported.

\section{ACKNOWLEDGMENTS}

This work was supported by Youngsan University Research Fund of 2019.

\section{REFERENCES}

Anderson SD, Kippelen P. Airway injury as a mechanism for exercise-induced bronchoconstriction in elite athletes. J Allergy Clin Immunol 2008;122:225-235.

Coyle EF, Montain SJ. Benefits of fluid replacement with carbohydrate during exercise. Med Sci Sports Exerc 1992;24(9 Suppl):S324-330.

Daviskas E, Gonda I, Anderson SD. Local airway heat and water vapour losses. Respir Physiol 1991;84:115-132.

Daviskas E, Gonda I, Anderson SD. Mathematical modeling of heat and water transport in human respiratory tract. J Appl Physiol (1985) 1990; 69:362-372.

de Oliveira EP, Burini RC. The impact of physical exercise on the gastrointestinal tract. Curr Opin Clin Nutr Metab Care 2009;12:533-538.

Hiller WD. Dehydration and hyponatremia during triathlons. Med Sci Sports Exerc 1989;21(5 Suppl):S219-221.

Jeukendrup AE, Jentjens RL, Moseley L. Nutritional considerations in triathlon. Sports Med 2005;35:163-181.

Kalhoff H. Mild dehydration: a risk factor of broncho-pulmonary disorders? Eur J Clin Nutr 2003;57 Suppl 2:S81-87.

Kim $\mathrm{CH}$, Kwak YS. Swim training increases ovalbumin induced active systemic anaphylaxis in mice. Immunol Invest 2004;33:469-480.

Kim CW, Figueroa A, Park CH, Kwak YS, Kim KB, Seo DY, Lee HR. Combined effects of food and exercise on anaphylaxis. Nutr Res Pract 2013; 7:347-351.

Park CH, Kim KB, Han J, Ji JG, Kwak YS. Cardiac damage biomarkers following a triathlon in elite and non-elite triathletes. Korean J Physiol Pharmacol 2014;18:419-423.

Park CH, Kwak YS. Changes of cardiac biomarkers after ultradistance and standard-distance triathlon. J Exerc Rehabil 2019;15:254-257.

Pichurko BM, McFadden ER Jr, Bowman HF, Solway J, Burns S, Dowling $\mathrm{N}$. Influence of cromolyn sodium on airway temperature in normal subjects. Am Rev Respir Dis 1984;130:1002-1005.

Qamar MI, Read AE. Effects of exercise on mesenteric blood flow in man. Gut 1987;28:583-587.

Rundell KW, Anderson SD, Sue-Chu M, Bougault V, Boulet LP. Air quality and temperature effects on exercise-induced bronchoconstriction. Compr Physiol 2015;5:579-610.

Simpson AJ, Romer LM, Kippelen P. Exercise-induced dehydration alters pulmonary function but does not modify airway responsiveness to 
dry air in athletes with mild asthma. J Appl Physiol (1985) 2017;122: 1329-1335

Van Deventer S, Gouma D. Bacterial translocation and endotoxin transmigration in intestinal ischaemia and reperfusion. Curr Opinion
Aneasth 1994;7:126-130.

Van Nieuwenhoven MA, Vriens BE, Brummer RJ, Brouns F. Effect of dehydration on gastrointestinal function at rest and during exercise in humans. Eur J Appl Physiol 2000;83:578-584. 